\title{
Universiteit
}

Leiden

The Netherlands

\section{Natural deep eutectic solvents as biofilm structural breakers}

Nava-Ocampo, M.F.; Al Fuhaid, L.; Verpoorte, R.; Choi, Y.H.; Loosdrecht, M.C.M. van;

Vrouwenvelder, J.S.; ... ; Bucs, S.S.

\section{Citation}

Nava-Ocampo, M. F., Al Fuhaid, L., Verpoorte, R., Choi, Y. H., Loosdrecht, M. C. M. van, Vrouwenvelder, J. S., ... Bucs, S. S. (2021). Natural deep eutectic solvents as biofilm structural breakers. Water Research, 201. doi:10.1016/j.watres.2021.117323

Version: $\quad$ Publisher's Version

License: $\quad$ Licensed under Article 25fa Copyright Act/Law (Amendment Taverne)

Downloaded from: https://hdl.handle.net/1887/3248764

Note: To cite this publication please use the final published version (if applicable). 


\title{
Natural deep eutectic solvents as biofilm structural breakers
}

\author{
Szilárd S. Bucs ${ }^{\text {a }}$ \\ ${ }^{\text {a } W a t e r ~ D e s a l i n a t i o n ~ a n d ~ R e u s e ~ C e n t e r, ~ K i n g ~ A b d u l l a h ~ U n i v e r s i t y ~ o f ~ S c i e n c e ~ a n d ~ T e c h n o l o g y, ~ T h u w a l, ~ S a u d i ~ A r a b i a ~}$ \\ ${ }^{\mathrm{b}}$ Natural Products Laboratory, Institute Biology Leiden, Leiden University, Leiden, the Netherlands \\ ${ }^{\mathrm{c}}$ Biochemical Engineering, Delft University of Technology, Julianalaan 67, 2628 BC Delft, the Netherlands
}

Maria F. Nava-Ocampo ${ }^{\mathrm{a}}$, Lamya Al Fuhaid ${ }^{\mathrm{a}}$, Robert Verpoorte ${ }^{\mathrm{b}}$, Young Hae Choi ${ }^{\mathrm{b}}$, Mark C. M. van Loosdrecht ${ }^{c}$, Johannes S. Vrouwenvelder ${ }^{a}$, Geert J. Witkamp ${ }^{a}$, Andreia S.F. Farinha ${ }^{\text {a, }}$,

\section{A R T I C L E I N F O}

\section{Keywords:}

Natural Deep Eutectic Solvents

biofilm structural modifiers

green solvents

aerobic granular sludge

\begin{abstract}
A B S T R A C T
Natural Deep Eutectic Solvents (NADES) are composed of supramolecular interactions of two or more natural compounds, such as organic acids, sugars, and amino acids, and they are being used as a new media alternative to conventional solvents. In this study, a new application of NADES is presented as a possible technology for biofilm structural breaker in complex systems since the current solvents used for biofilm cleaning and extraction of biofilm components use hazardous solutions. The NADES (betaine:urea:water and lactic acid:glucose:water) were analyzed before and after the biofilm treatment by attenuated total reflection Fourier-transform infrared spectroscopy and fluorescence excitation-emission matrix spectroscopy. Our results indicate that the green solvents could solubilize up to $\approx 70$ percent of the main components of the biofilms extracellular matrix. The solubilization of the biomolecules weakened the biofilm structure, which could enhance the biofilm solubilization and removal. The NADES have the potential to be an environment-friendly, green solvent to extract valuable compounds and break the main structure from the biofilm, leading to a greener method for extracellular polymeric substance (EPS) extraction and biofilm treatment in various water treatment systems.
\end{abstract}

\section{Introduction}

Natural deep eutectic solvents (NADES) have been extensively studied to develop and find green organic solvents that can be used in a big variety of industries such as water treatment. NADES are mixtures of natural compounds such as amino acids, sugars, sugar alcohols, polyalcohols, and organic acids and bases that interact through hydrogen bonding (Choi et al., 2011; Dai et al., 2016, 2013a; Vanda et al., 2018). The fact that the individual components of NADES are natural compounds makes these green solvents biodegradable, harmless, and environmentally friendly, compared to most of their predecessors, ionic liquids and deep eutectic solvents (Paiva et al., 2014).

NADES are characterized by having melting points significantly lower than their components and are abundant in nature (Paiva et al., 2014). The viscosity of the NADES can be easily adjusted by increasing the water content maintaining the solvent properties (Dai et al., 2013a, 2015). These green solvents have been used during the past decade in diverse applications such as: i) media for enzymatic or chemical reactions to solubilize or isolate macromolecules (Ma et al., 2020), ii) alternative for non-water-soluble drugs (Liu et al., 2018), iii) to develop cosmetic ingredients (Paiva et al., 2014), iv) for extraction of bioactive metabolites from plants (Dai et al., 2013b; Gonzalez et al., 2018), and v) biomedical applications (Plotka-Wasylka et al., 2017; Vanda et al., 2018). Nevertheless, there is still much to learn about the possible applications of the NADES.

Among the applications of NADES is the extraction of biopolymers. Previously, NADES have been actively employed for the extraction of biomolecules from several biomass sources, including lignocellulosic biomass rice straw (Kumar et al., 2016), date palm biomass (Fang et al., 2017), and corncob biomass (Zhang et al., 2012). Li et al. (Li et al., 2016) utilized a betaine-based NADES for protein extraction from calf blood samples; Lim et al. (Lim et al., 2017) studied the potential of the detachment of Streptococcus mutans from wells, which occurs by partial dissolution of exopolysaccharides that surround the biofilm. While

\footnotetext{
Abbreviations: Natural deep eutectic solvents, (NADES); Fourier-transform infrared spectroscopy, (ATR-FTIR); Fluorescence excitation-emission matrix spec-

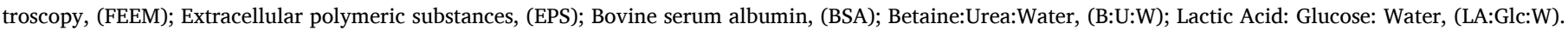

* Corresponding author.

E-mail address: andreia.farinha@kaust.edu.sa (A.S.F. Farinha).
} 
Table 1

NADES components and composition.

\begin{tabular}{|c|c|c|c|c|}
\hline Component 1 & Component 2 & Component 3 & $\begin{array}{l}\text { Molar } \\
\text { ratio }\end{array}$ & Abbreviation \\
\hline Urea & Ethylene glycol & Water & 10:10:1 & U:EG \\
\hline Lactic acid & Glucose & Water & $5: 1: 3$ & LA:Glc:W \\
\hline Lactic acid & $\begin{array}{l}\text { Choline } \\
\text { chloride }\end{array}$ & Water & $5: 1: 3$ & LA:ChCl \\
\hline Urea & $\begin{array}{l}\text { Choline } \\
\text { chloride }\end{array}$ & Water & $1: 1: 1$ & $\mathrm{U}: \mathrm{ChCl}$ \\
\hline $\begin{array}{c}\text { Ethylene } \\
\text { glycol }\end{array}$ & $\begin{array}{l}\text { Choline } \\
\text { chloride }\end{array}$ & Water & 1:2:0.1 & EG:ChCl \\
\hline Betaine & Urea & Water & 1:1:10 & B:U:W \\
\hline Betaine & Urea & Water & $2: 1: 10$ & 2B:U:W \\
\hline
\end{tabular}

previous studies have shown that NADES can be successfully used for macromolecules extraction, there is limited information about the use of NADES to extract proteins and polysaccharides from polyculture biofilms and even less information about their potential for biofilm removal. In specific industries such as food, medicine, paper, and water treatment, biofilm growth generates economic losses and hinders production (Hall-Stoodley et al., 2004; Lim et al., 2017).

A biofilm is formed by a group of microorganisms surrounded by extracellular polymeric substances (EPS) to protect themselves from hostile environments. The EPS is also involved in the biofilm attachment to the surface and prevents detachment (Flemming, 2020; Lim et al., 2017; Limoli et al., 2015). Therefore, biofilm treatment could be achieved successfully by focusing on the disruption of the EPS matrix.

Current curative (cleaning) and preventive strategies for biofilm control applied in diverse industries have failed to remove the biofilm from the surfaces entirely, and the use of chemicals creates environmental concerns (Creber et al., 2010). In the particular case of desalination by reverse osmosis, the amount of chemical used and cleaning frequency depend on the feed water quality, membrane type, and the number of elements in the desalination train. For example, a full-scale operation plant in Utrecht, Netherlands having river water as feedwater, with spiral polyamide membrane modules of $2.5^{\prime \prime} \mathrm{X} 40^{\prime \prime}$, and a cleaning frequency of 17 times per year with sodium hydroxide and hydrochloric acid, use $0.8 \mathrm{~m}^{3}$ of chemical cleaning per membrane area (Beyer et al., 2017).

This study evaluates seven different NADES as potential cleaning agents for biofilm removal by dissolving or breaking the biofilm structure. Aerobic granular sludge (AGS) is used as a model biofilm since the granules' weight and form can be observed and measured, allowing the results to be precise and reproducible. Also, the use of NADES to treat AGS can give some insights into the application of these green solvents for EPS extraction and destabilization of the biofilm structure, overcoming the use of harsh chemicals currently used for EPS extraction, such as formaldehyde and $\mathrm{NaOH}$ (Farhat et al., 2019; Feng et al., 2019). This study proposed a greener alternative to the current solvents used for EPS extraction and purification, as well as biofouling treatment in various water treatment systems.
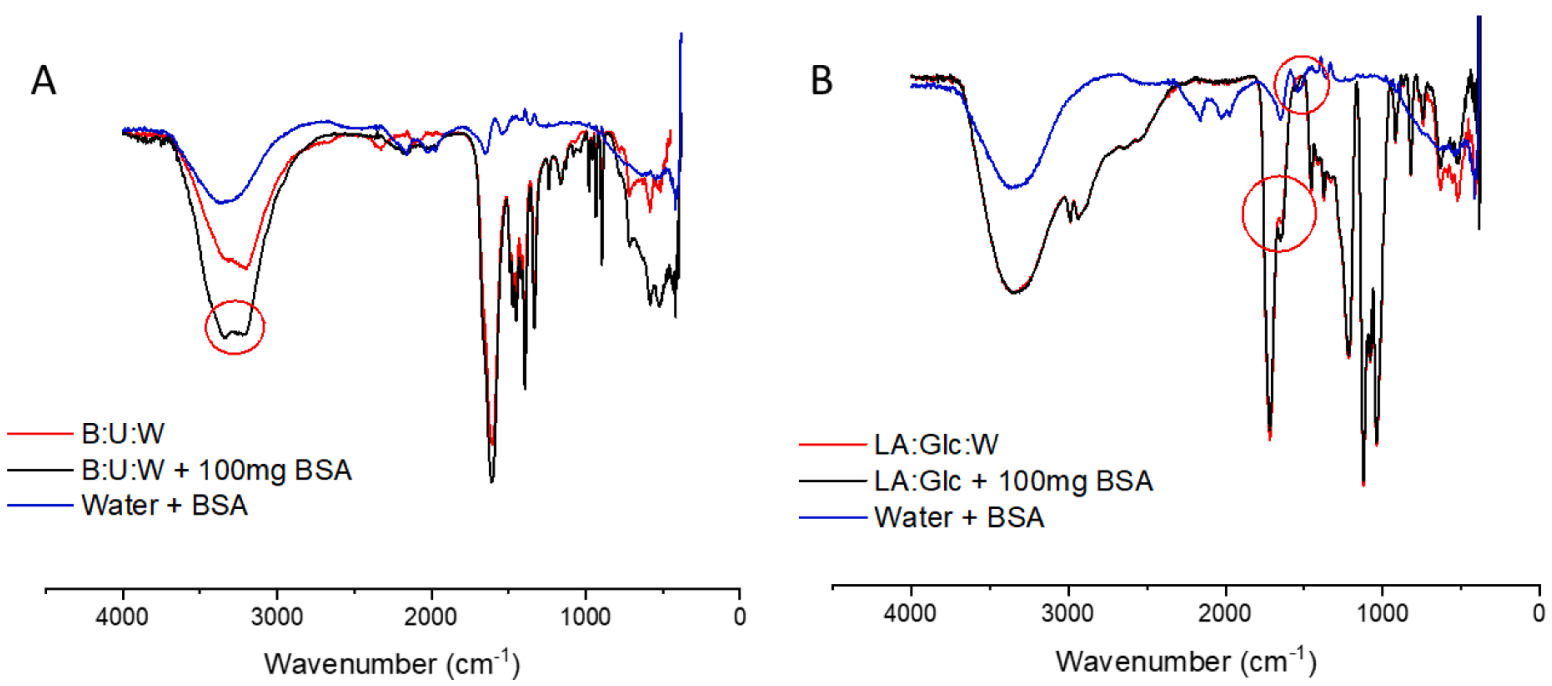

Fig. 1. ATR-FTIR spectra of the interaction of Bovine Serum Albumin (BSA) with Betaine:Urea:Water (1:1:10) (A), and Lactic Acid:Glucose:Water (5:1:3) (B). Each ATR-FTIR shows the comparison of the pure NADES (red), $100 \mathrm{mg}$ BSA fully dissolved in the solution of each NADES (black), and $100 \mathrm{mg}$ of BSA in DI water (blue), illustrating the presence of BSA on the NADES.
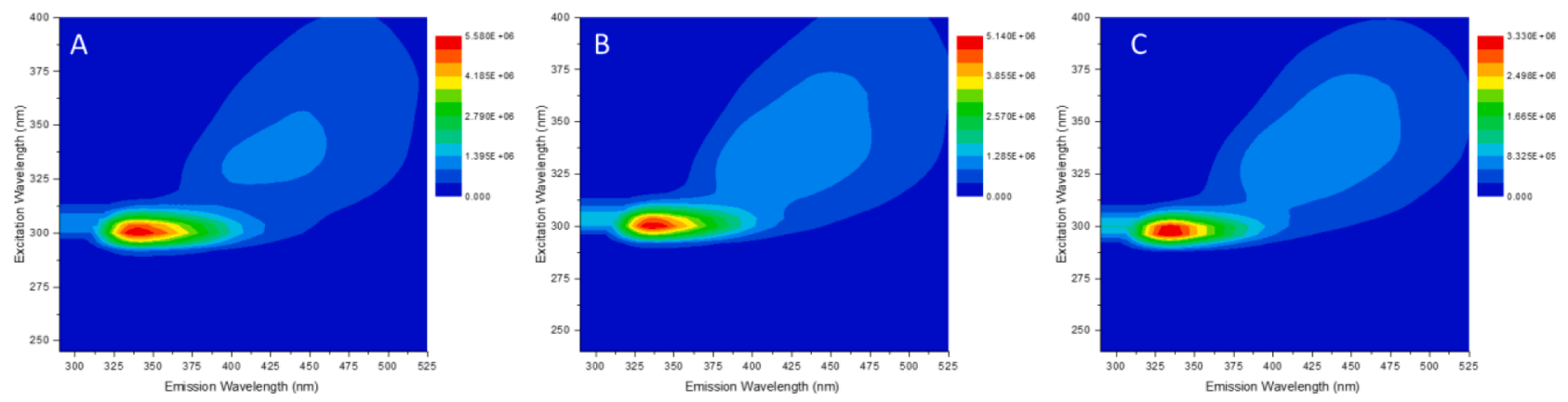

Fig. 2. FEEM of the BSA presence with deionized (DI) water (control) (A), Betaine:Urea:Water (1:1:10) (B), and Lactic Acid:Glucose:Water (5:1:3) (C), illustrating the solubility potential of BSA in each of the NADES. 

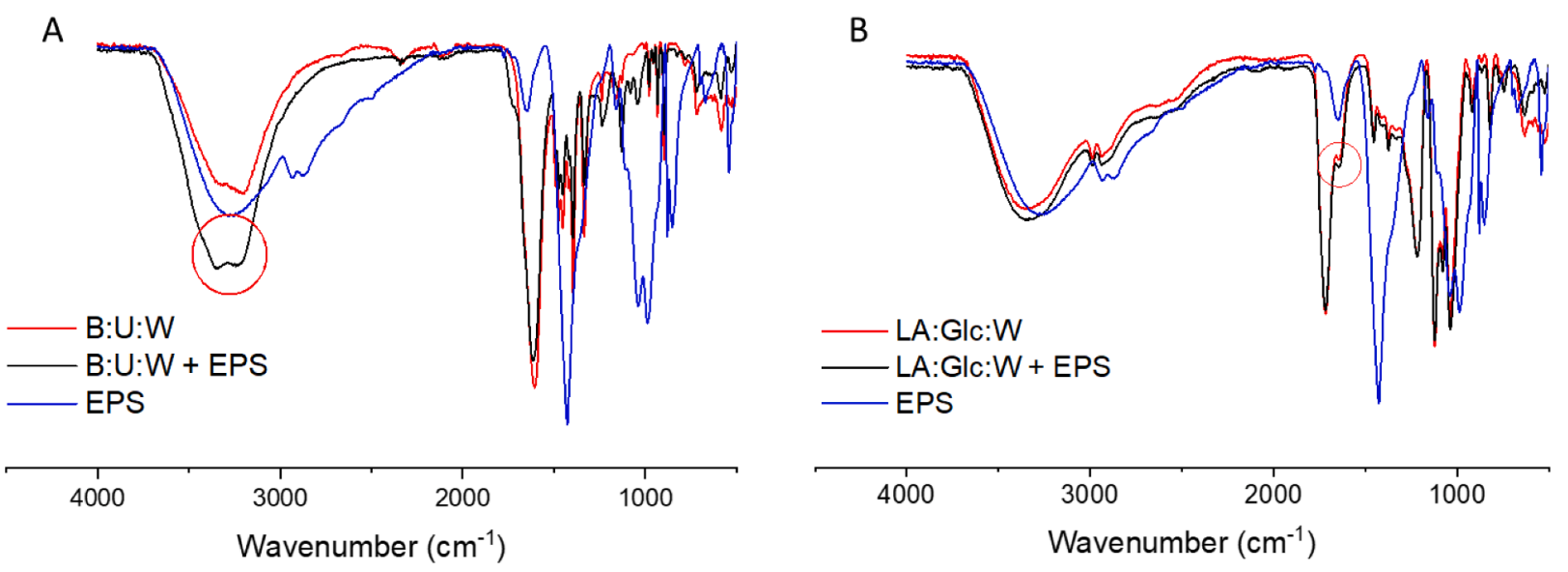

Fig. 3. ATR-FTIR spectra of the interaction of EPS with Betaine:Urea:Water (1:1:10) (A), and Lactic Acid:Glucose:Water (5:1:3) (B). Each ATR-FTIR shows the comparison of the pure NADES (red), NADES with EPS extracted from a biofilm that was dissolved in NADES (black), and the ATR-FTIR of the solid EPS extracted from a biofilm (blue). Both spectra indicate that the EPS is present in the NADES.
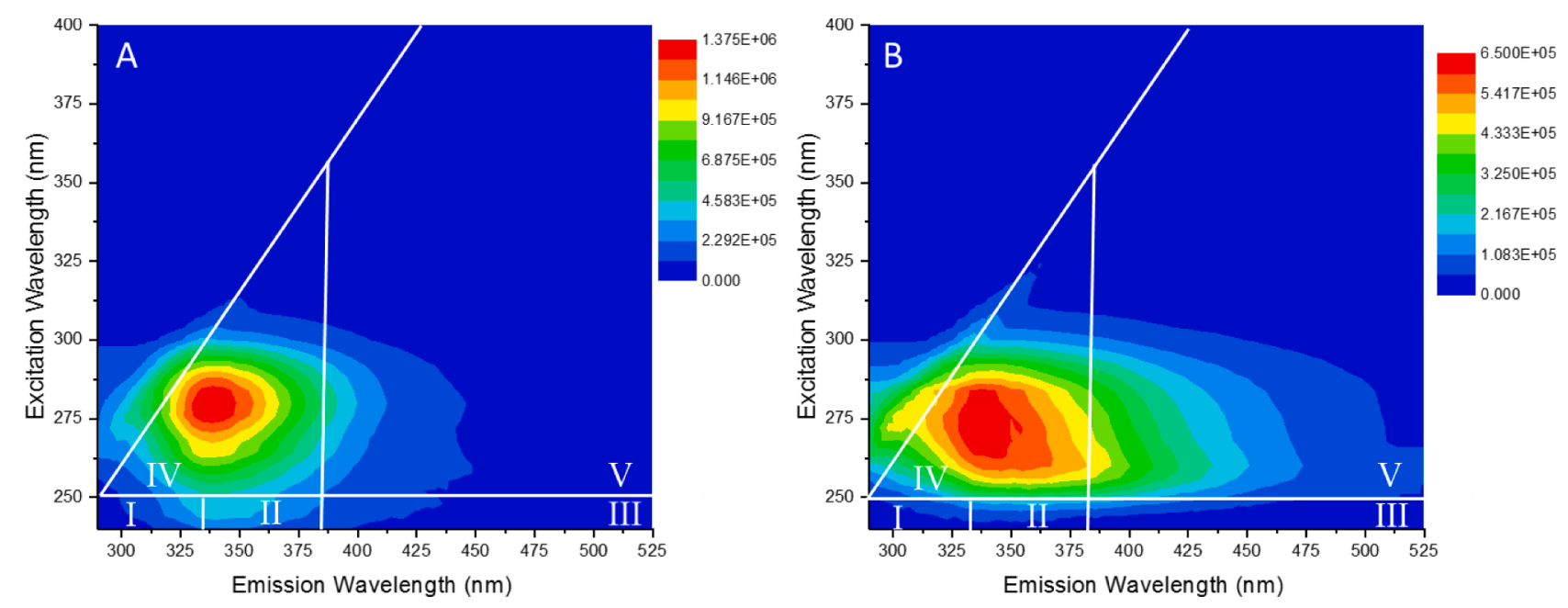

Fig. 4. FEEM of biofilm extracted EPS with Betaine:Urea:Water (1:1:10) (A), and Lactic Acid:Glucose:Water (5:1:3) (B). Indicated with white lines, regions I and II correspond to aromatic protein-like compounds, region III to fluvic acid-like compounds, region IV to microbial like compounds (e.g.,. tryptophan and protein-like), and region V to humic-acid like compounds (Chen et al., 2003; Zhou et al., 2013). The high peak in region IV indicates the presence of EPS proteins in the NADES.
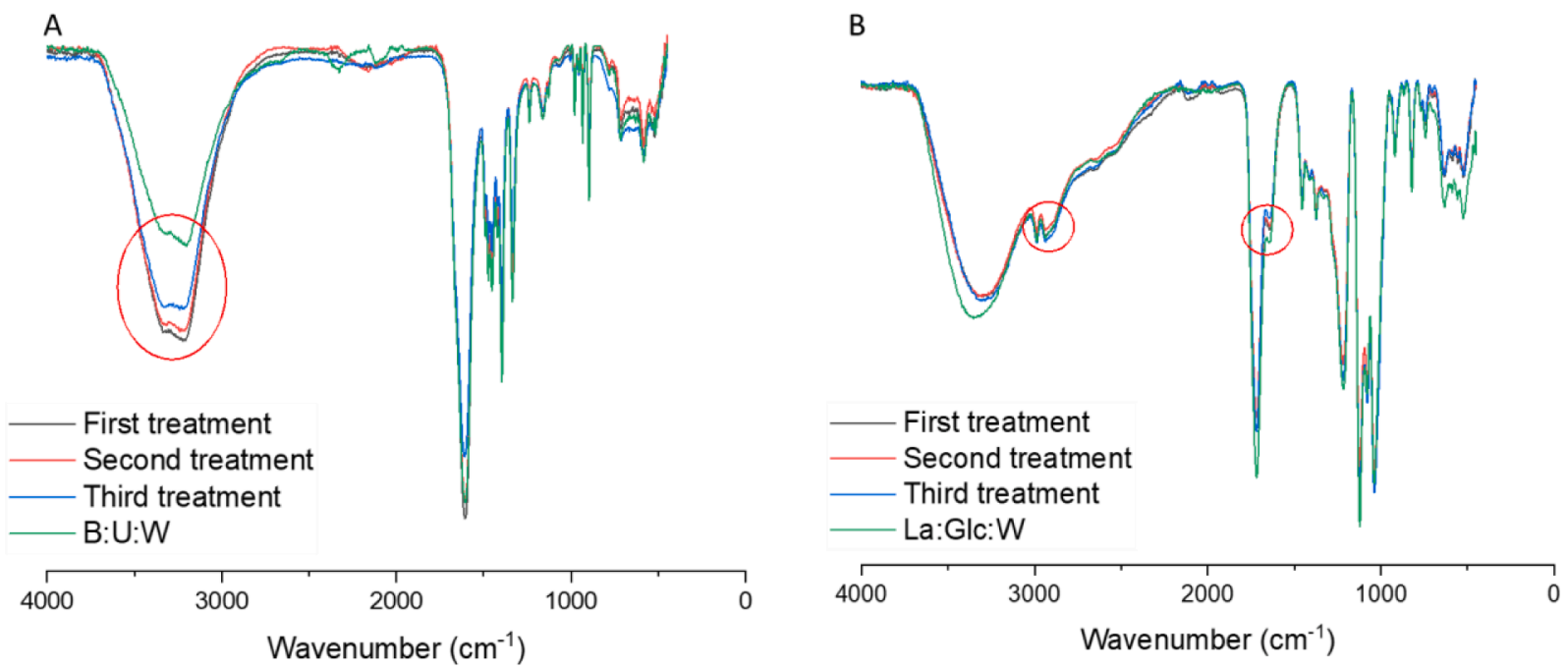

Fig. 5. ATR-FTIR of the NADES after treating lab-grown granules. Betaine:Urea:Water (1:1:10) (A), and Lactic Acid:Glucose:Water (5:1:3) (B), indicating solubilization of the lab-grown granules components. 

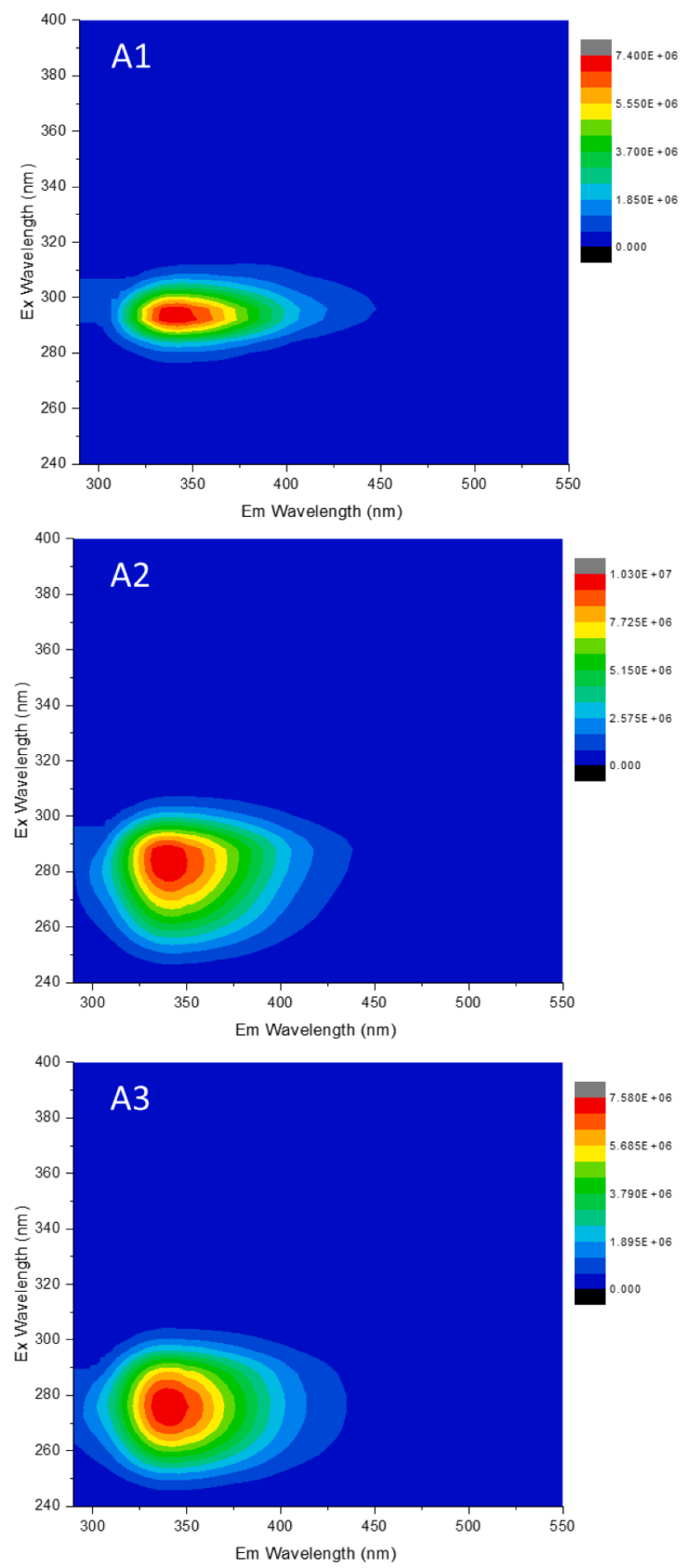
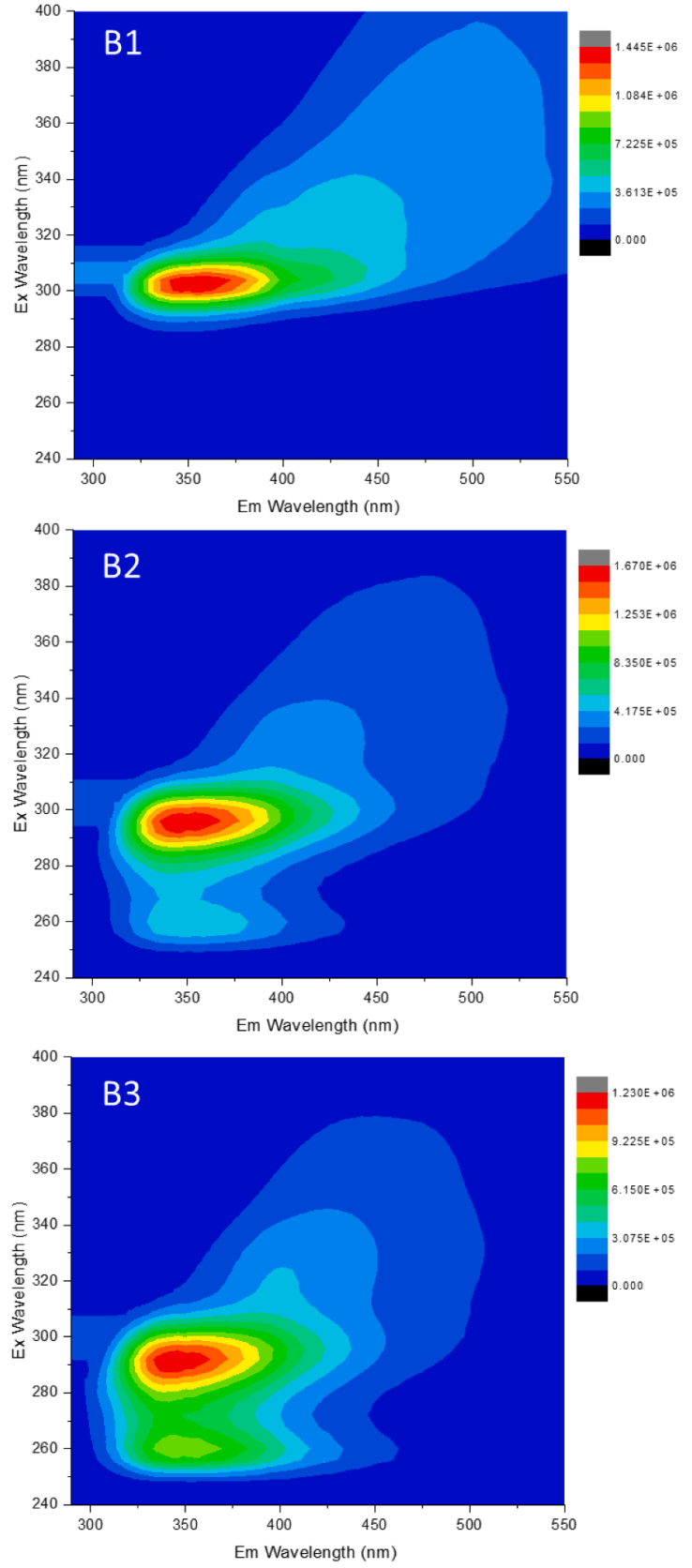

Fig. 6. FEEM of the NADES after the treatment of pilot-plant granules, after first, second and third treatment with Betaine:Urea:Water (1:1:10), A1, A2, A3, and with Lactic Acid:Glucose:Water (5:1:3), B1, B2, and B3. Indicating the solubilization of proteins that are part of the lab-grown granules matrix.

\section{Materials and methods}

\subsection{Natural deep eutectic solvents}

All NADES used for this study were prepared at $50^{\circ} \mathrm{C}$ in a water bath until a transparent solution was obtained at the molar ratios specified in Table 1. Urea, ethylene glycol, lactic acid, glucose, choline chloride, and betaine were purchased from Sigma-Aldrich (St. Louis, Missouri, USA) and used without further purification. All the NADES showed to be stable at room temperature for more than four months, except for Urea: Ethylene glycol, which precipitated after eight hours at room temperature $\left(23^{\circ} \mathrm{C}\right)$. After a screening of these seven NADES, B:U:W and LA:Glc: $\mathrm{W}$ were selected for the follow-up studies described in this paper because of their capacity to solubilize bovine serum albumin (BSA).

\subsection{Protein solubility in NADES}

The solubility of BSA, as a model protein, was tested using the seven different NADES. Since the BSA solubility in water is $40 \mathrm{mg} \cdot \mathrm{ml}^{-1}$, three milliliters of each NADES was added to $100 \mathrm{mg}$ of BSA. All samples were kept at $40{ }^{\circ} \mathrm{C}$ with constant shaking at $120 \mathrm{rpm}$ for $24 \mathrm{~h}$. The NADES that fully dissolved the BSA (visual inspection) and in which no solid residual was observed were analyzed by attenuated total reflection FourierTransform Infrared spectroscopy (ATR-FTIR) (PerkinElmer Inc., Waltham, Massachusetts, USA) and Fluorescence Excitation Emission Matrix (FEEM) (Spectrofluorometer, Horiba) spectroscopy.

\subsection{Extracellular polymeric substances of biofilm solubility in NADES}

The solubility of EPS was tested in NADES. Two $\mathrm{mL}$ of each NADES was added to $5 \mathrm{mg}$ of EPS previously extracted from a membrane biofilm 

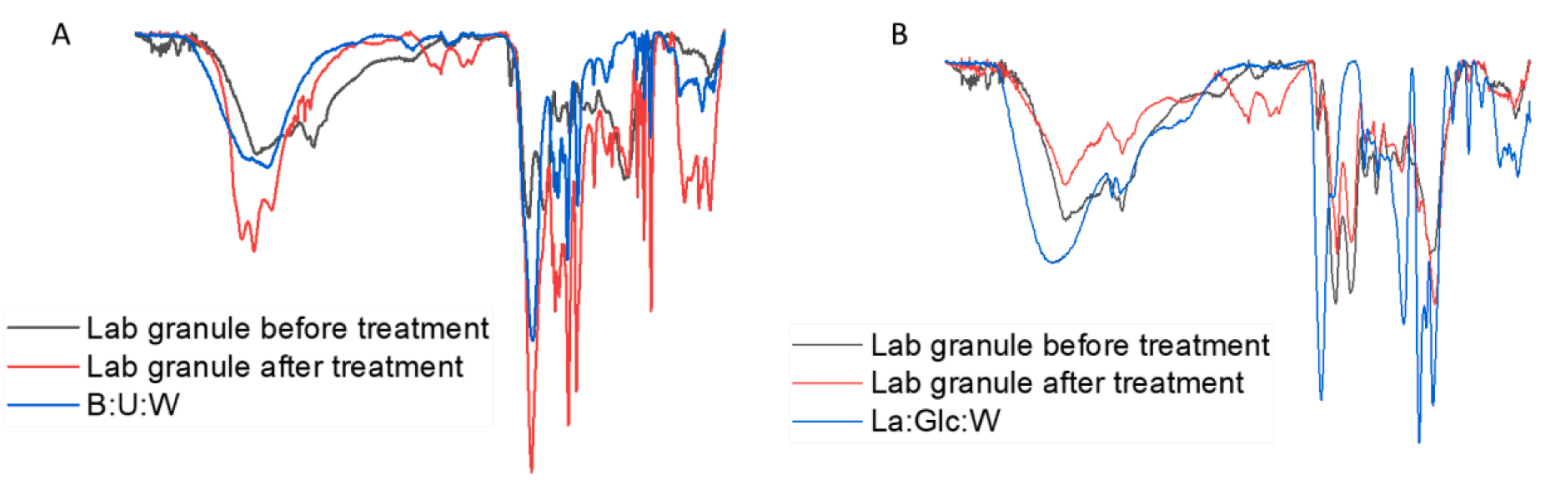

\begin{tabular}{ccccc}
\hline 4000 & 3000 & 2000 & 1000 & 0 \\
& Wavenumber $\left(\mathrm{cm}^{-1}\right)$ & &
\end{tabular}

$\begin{array}{cccc}4000 & 3000 & 2000 & 1000\end{array}$

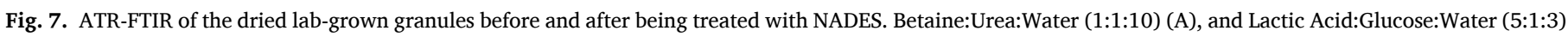
(B). The ATR-FTIR spectra show a strong presence of B:U:W in the granule after being treated.

grown in a membrane fouling simulator (Vrouwenvelder et al., 2007). The EPS was extracted following the formaldehyde- $\mathrm{NaOH}$ protocol (Feng et al., 2019). All samples were kept at $40{ }^{\circ} \mathrm{C}$ and continuously shaken at $120 \mathrm{rpm}$ for $24 \mathrm{~h}$.

\subsection{Lab granules}

Aerobic granules were collected from a lab-scale bioreactor (Figure S1A). The NADES potential to solubilize the granules components (e.g., proteins) was analyzed. For each NADES (B:U:W and LA:Glc: $\mathrm{W}), 2 \mathrm{~g}$ of granules (wet weight) were freeze-dried to remove all water content that could dilute the NADES and submerged in $10 \mathrm{~mL}$ of each NADES. Once each NADES was added to the granule, the samples were kept at $40{ }^{\circ} \mathrm{C}$ at $120 \mathrm{rpm}$ for $24 \mathrm{~h}$. The $40{ }^{\circ} \mathrm{C}$ temperature was chosen because it is the temperature commonly used for biofouling treatment and to enhance the granule-NADES interaction. The granules were filtered, and $10 \mathrm{~mL}$ of fresh NADES was added to the granules' remainings. The NADES treatment was repeated three times. After each treatment, the NADES was removed and analyzed by ATR-FTIR and FEEM to analyze changes in the NADES composition. The remaining granules were washed three times with deionized (DI) water to remove the NADES residual from the biofilm. Finally, the granules were freezedried and analyzed by ATR-FTIR, and their structure was observed under the microscope (Stereomicroscope, Nikon SMZ 25).

\subsection{Pilot-plant granules}

Aerobic granules were collected from a pilot-plant located in Utrecht (The Netherlands), from the mixed liquor around 1 meter below the surface of the bioreactor (Figure S1B). The NADES potential to solubilize the granules' components (e.g., proteins) was analyzed. For each NADES (B:U:W and LA:Glc:W), $2 \mathrm{~g}$ of granules (wet weight) were freeze-dried, and $10 \mathrm{~mL}$ of each NADES was added. Once the granules were added to the NADES, the samples were kept at $40^{\circ} \mathrm{C}$ at $120 \mathrm{rpm}$ for $24 \mathrm{~h}$. After the NADES treatment, the supernatant was removed, and the remaining granules were treated again with fresh NADES. Since the granules were taken from a pilot-plant, the EPS matrix contains a large variety of compounds, e.g., humic and fluvic acids and various minerals, besides the extracellular proteins and polysaccharides. Therefore, the treatment of the pilot-plant granules with NADES was repeated eight times for each sample. After the eighth treatment, the granules' remainings were washed three times with DI water to remove the NADES residual from the biofilm. After each treatment, the NADES was removed and analyzed by ATR-FTIR and FEEM.

\section{Results}

\subsection{BSA solubility in NADES}

In this study, we tested seven different NADES (Table 1). Based on visual observations (Figure S2), B:U:W in a molar ratio of (1:1:10) and LA:Glc:W in a molar ratio of (5:1:3) successfully solubilized $100 \mathrm{mg}$ of BSA. BSA in solid form was visible in the other NADES.

B:U:W and LA:Glc:W with BSA were analyzed by ATR-FTIR (Fig. 1) to confirm the presence of the BSA in the NADES. ATR-FTIR spectrum of B: U:W with BSA (Fig. 1A) showed an intensity increase in the bands in the region between 3000 and $3500 \mathrm{~cm}^{-1}$, which is attributed to the $\mathrm{O}-\mathrm{H}$ stretching, meaning an increase in the hydrogen bonds between the BSA and the B:U:W. In the case of LA:Glc:W and BSA (Fig. 1B), a band is observed at the wavenumber $1547 \mathrm{~cm}^{-1}$, and an increase is perceived at the wavenumber $1652 \mathrm{~cm}^{-1}$, which can be attributed to the addition of BSA. The variations in these bands observed in both NADES spectra confirm the presence of BSA moiety in NADES.

A comparison between BSA dissolved in water and BSA dissolved with each of the NADES was made by FEEM analysis (Fig. 2). The intrinsic fluorescence of BSA is observed in the water-BSA sample (Fig. 2A), which can be compared with the B:U:W-BSA (Fig. 2B) and LA: Glc:W-BSA (Fig. 2C). For both NADES, a confirmation of the BSA presence is observed by obtaining similar spectra as the one with water-BSA (BSA: Ex. 280 nm, Em. 300) (Wani et al., 2017) with a slight decrease in the fluorescence intensity.

\subsection{Extracellular polymeric substance solubility in NADES}

To analyze the biofilm solubilization potential of the two NADES, EPS was extracted from a membrane biofilm grown in membrane fouling simulators (MFS) (Vrouwenvelder et al., 2007). These MFS were run for seven days, and the biofilm was collected for EPS extraction, following formaldehyde- $\mathrm{NaOH}$ protocol (Farhat et al., 2019; Feng et al., 2019). The extracted EPS was placed in B:U:W and LA:Glc:W. After $24 \mathrm{~h}$, a visually homogeneous and transparent solution was obtained in both cases. The solution containing B:U:W and the biofilm EPS (Fig. 3A) showed an increase in the bands' intensity in the region between 3000 and $3500 \mathrm{~cm}^{-1}$, also observed in the case of the BSA (Fig. 1A). This increase is attributed to $\mathrm{O}-\mathrm{H}$ stretching, indicating an increase in the number of hydrogen bonds between the EPS and the B:U:W. For the interaction of biofilm EPS and LA:Glc:W (Fig. 3B), the same increase in the band $1652 \mathrm{~cm}^{-1}$ observed in the presence of BSA (Fig. 1B) is also seen here, which is attributed to the proteins present in the EPS.

To examine the predominant components of the extracted EPS from 


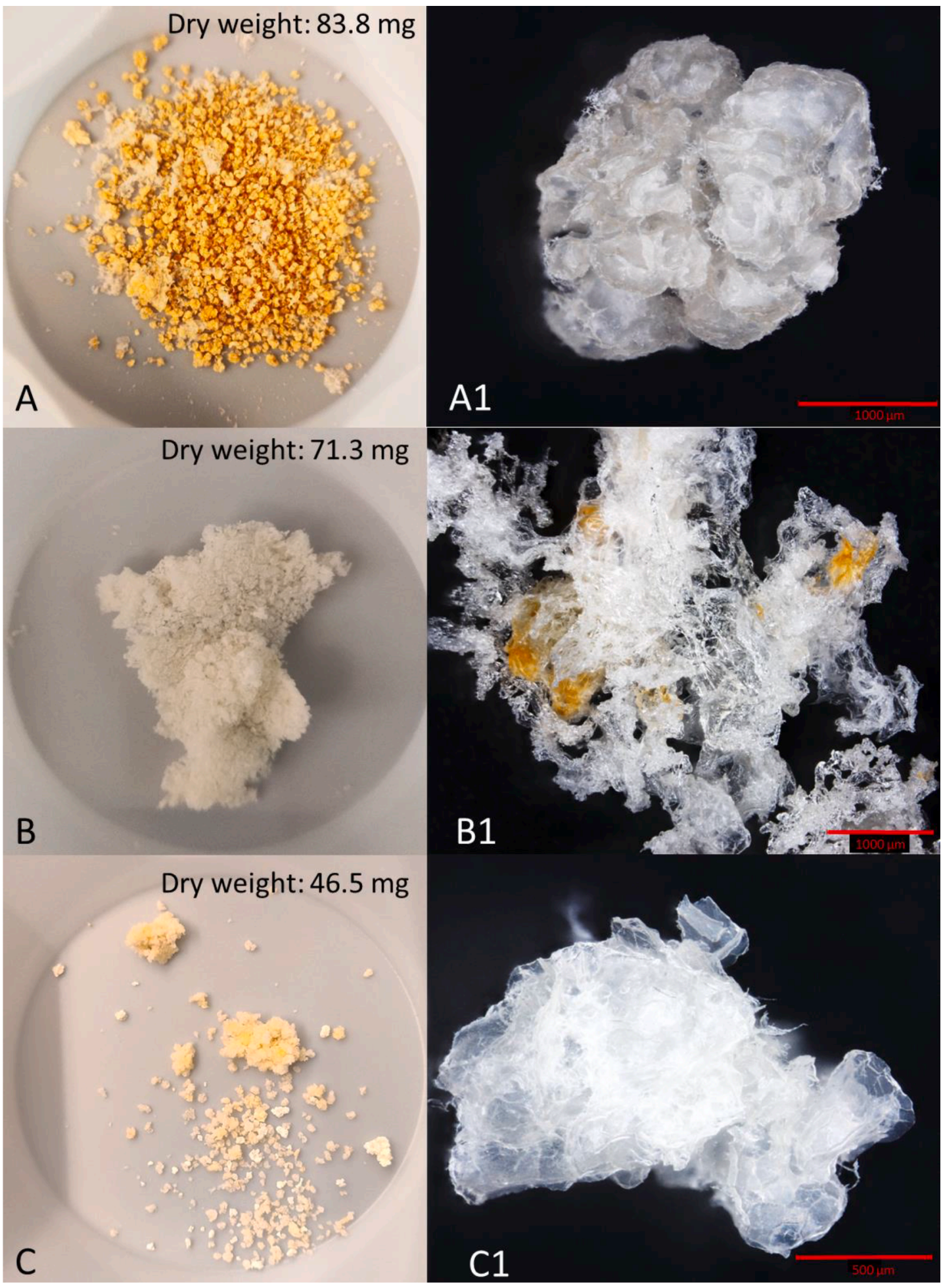

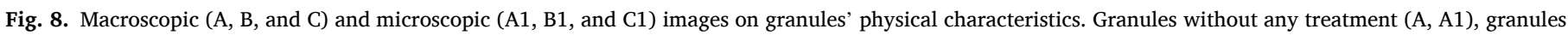

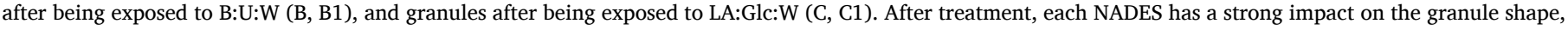
size, and structure.

biofilms, FEEM analysis was used. According to the classification by Zhou et al. (2013), the emission excitation spectra can be separated into five regions as follows: region I and II correspond to aromatic, protein-like compounds, region III to fluvic acid-like compounds, region IV to microbial-like compounds (e.g., tryptophan and protein-like) and region V to humic-acid like compounds (Chen et al., 2003; Zhou et al., 2013). The region classification of FEEM is typically used in water research due to the usual components found in biofilms (Sanawar et al., 2018; Zhou et al., 2013). After solubilizing the extracted EPS, both tested NADES (B:U:W, Fig. 4A and LA:Glc:W, Fig. 4B) show distinct peaks in the microbial-like compounds region (Region IV), which suggests that the used NADES can solubilize the proteins that are part of the EPS. 

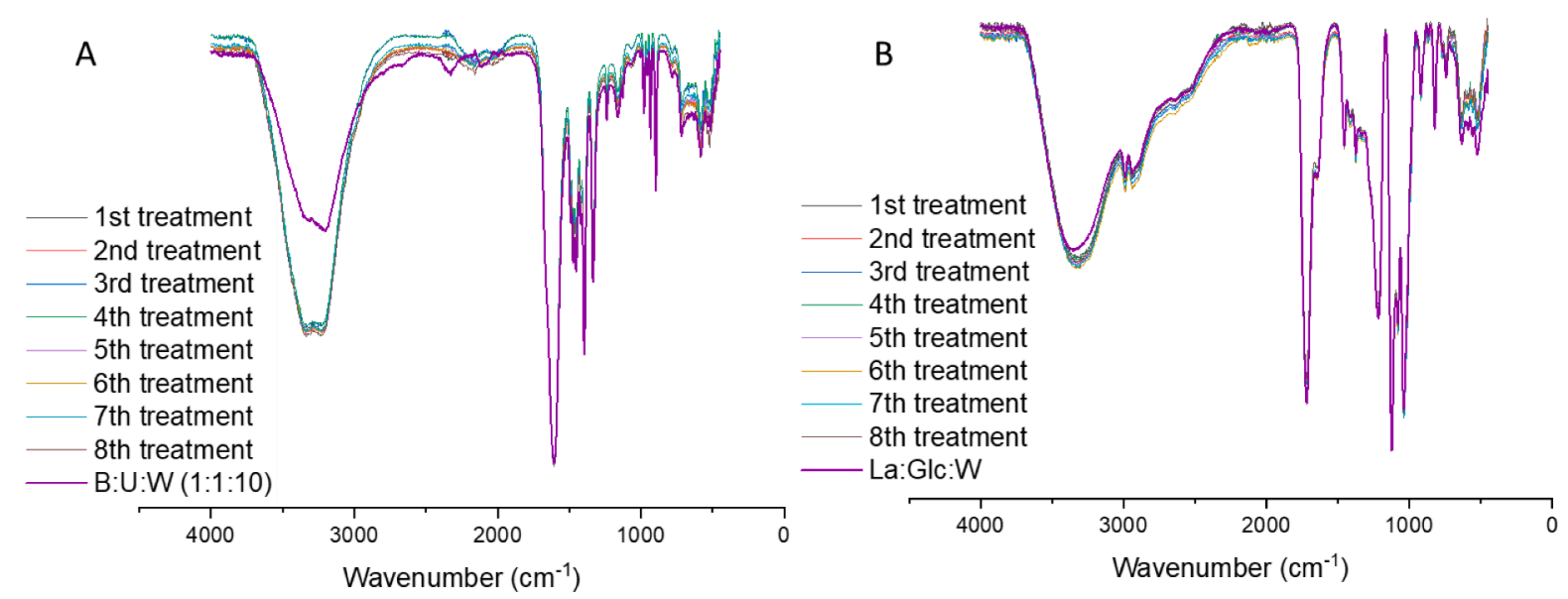

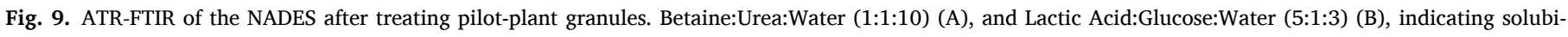
lization of the pilot-plant granules components.

\subsection{Aerobic granular sludge solubility in NADES}

The aerobic granular sludge (AGS) are dense self-immobilized microbial granules that have a compact microbial structure with high EPS content (Nancharaiah and Sarvajith, 2019). The EPS is a rich matrix of proteins, polysaccharides, glycoproteins, nucleic acids, phospholipids, and humic acids (McSwain et al., 2005; Nancharaiah and Sarvajith, 2019). To further analyze the biofilm solubilization potential of the NADES, AGS obtained from a lab-scale bioreactor were used as model biofilms. Before the addition of NADES to the granules, the AGS used for this study were freeze-dried to remove all water content within the granules. This step is needed to ensure that the observed variations, mainly in the ATR-FTIR, are caused only by the solubilization of the AGS biomolecules and not by the addition of water into the NADES. After each NADES treatment, the samples were analyzed to confirm the presence of the biomolecules in the NADES. Based on the results obtained from the ATR-FTIR spectra, the increase in the bands between 3000 and $3500 \mathrm{~cm}^{-1}$ can be attributed to $\mathrm{O}-\mathrm{H}$ stretching as a result of an increase in the number of hydrogen bonds between the laboratory AGS and the B:U:W (Fig. 5A). It can also be observed that there is a variation in the amount of extracted biomolecules between the first, second, and third B:U:W treatment. For the LA:Glc:W (Fig. 5B), the ATR-FTIR of the granule does not seem to interfere with the LA:Glc:W peaks, except for a mild sharpness in the band $1652 \mathrm{~cm}^{-1}$, which can be seen in Figs. 1 and 3 with the BSA and EPS, respectively.

The FEEM plot (Fig. 6) confirmed that the macromolecules of AGS, such as proteins, are being solubilized within the NADES. After the first treatment with B:U:W (Fig. 6A1) and LA:Glc:W (Fig. 6B1), the plot shows distinct peaks related to proteins with similar characteristics as the ones found in BSA. After the second and third treatment with B:U:W (Fig. 6A2, B3) and LA:Glc:W (Fig. 6B2, B3) it is visible that the NADES is solubilizing the microbial by-products-like compounds (Region IV), which suggests that NADES can solubilize the proteins that are part of a biofilm matrix. This result is in concordance with other studies that utilized NADES to extract proteins and structural polymers from variable bio-sources like Streptococcus mutans, biomass from rice-straw, and calf blood samples (Kumar et al., 2016; Li et al., 2016; Lim et al., 2017). By comparing the three consecutive treatments by FEEM, it can be observed that the fluorescent peaks of the second and third treatment with B:U:W shifted more into the microbial by-products-like region of the FEEM compared with LA:Glc:W. Therefore, it seems that B:U:W can reach the granular components at a deeper level, and therefore, has a greater impact on the granules' structure.

To observe the effect of the NADES in the solid structure of the granules, the granules were washed with DI water three times to remove the NADES residuals, followed by freeze-drying. The granules were analyzed by ATR-FTIR, macroscopic visualization, and dry-weight. The $\mathrm{B}: \mathrm{U}: \mathrm{W}$ treatment on the solid granule residue (Fig. 7A), seemed to point at a more significant impact on the granule structure than the treatment with the LA:Glc:W (Fig. 7B). The solid granule residue after treatment with B:U:W ATR-FTIR spectrum showed a higher similarity to the B:U:W NADES ATR-FTIR spectrum, i.e., a strong band around $3000 \mathrm{~cm}^{-1}$ due to the $\mathrm{N}-\mathrm{H}$ and $\mathrm{O}-\mathrm{H}$ groups stretching, which suggests a high presence and intercalation of the green solvent components into the structure of the granules. Substracting the granule contribution in the band around $3000 \mathrm{~cm}^{-1}$ from the one corresponding to the granule after B:U:W treatment, we found that only $36 \%$ of the intensity belongs to the granule itself, meaning that $64 \%$ of the solid residuals is the intercalated NADES. For the LA:Glc:W, there is a negligible presence of NADES components (less than $1 \%$ in the bands around $3000 \mathrm{~cm}^{-1}$ ), but there is still a decrease in the intensity of the band around $3000 \mathrm{~cm}^{-1}$, compared to the non treated granule. This result suggests the removal of biomolecules from the moiety of the AGS.

The granules' form and shape before and after the NADES treatment were examined through macro and microscopic visualization (Fig. 8). There is a clear and strong impact on the granule shape, size, and structure after treatment with the NADES (B:U:W, Fig. 8B, B1, and LA: Glc:W, Fig. 8C, C1) compared with the non-treated granules (Fig. 8A, A1). The interaction between the granules and each NADES seemed different since there are physical variations in the granule macroscopic aspects after each NADES treatment. The treatment with B:U:W disrupted the shape and form of the granules entirely, while the treatment with LA:Glc:W decreased the size of the treated granules compared to the non-treated ones. Scanning electron microscopic images were taken which confirmed the disruption of the granular structure (Figure S3). Besides, there was a weight reduction of $69 \%$ in the granules treated with B:U:W based on the ATR-FTIR spectra, while the LA:Glc:W resulted in a $48 \%$ weight reduction. The result observed in Fig. $8 B 1$ and the granules ATR-FTIR spectra (Fig. 7A) suggest that the B:U:W NADES has a greater impact on the granule by breaking its structure and replacing the granule components with the B:U:W. These results confirm the solubilization of macromolecules (e.g., proteins and polysaccharides) by the green solvents used for treating biofilms.

To test a more realistic scenario, AGS obtained from a pilot-plant in Utrecht were treated with the selected NADES. The analysis made with ATR-FTIR of the B:U:W (Fig. 9A) resulted in similar spectra to the ones obtained with the laboratory-grown granules (Fig. 5A), only this time, we were not able to observe a difference between each of the NADES treatments. This could be attributed to the solubility limit of the NADES with the biomolecules present on the AGS. The spectra of the eight B:U: $\mathrm{W}$ treatments showed an increase mainly in the region between 3000 and $3500 \mathrm{~cm}^{-1}$, which can be attributed to various hydroxyl $\mathrm{O}-\mathrm{H}$ 

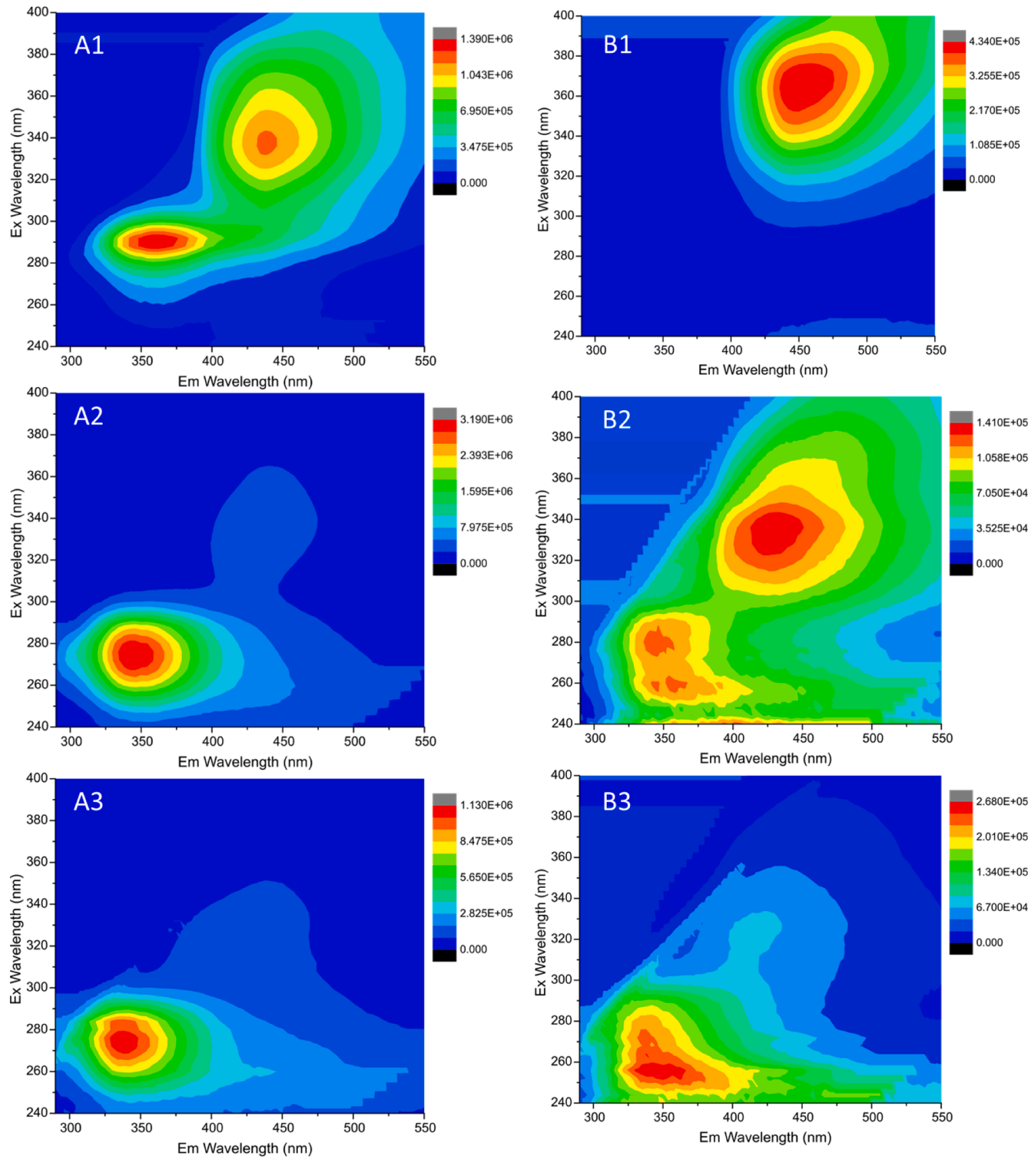

Fig. 10. FEEM of the NADES after the treatment of pilot-plant granules, after first, fourth and eighth treatment with Betaine:Urea:Water (1:1:10), A1, A2, A3, and with Lactic Acid:Glucose:Water (5:1:3), B1, B2, and B3. The NADES are capable to solubilize environmental components within the granules matrix.

groups stretchings (Fig. 9A). This increase indicates the intercalation of the components of the granules with the NADES. On the other hand, the spectra obtained of the NADES LA:Glc:W (Fig. 9B) after each treatment show a slight difference at the wavenumbers 1640 and $1720 \mathrm{~cm}^{-1}$, which can be attributed to the $\mathrm{N}-\mathrm{H}$ and $C=O$ stretchings, respectively, in the more complex system and components contained in pilot-plant AGS.

The potential of the NADES to solubilize more complex systems, such as pilot-plant granules, was also observed by FEEM (Fig. 10). Compared to the laboratory granules, the pilot-plant granules have more complex components, given the environment and treatment they have been exposed to. Therefore, the pilot-plant AGS were treated with the green solvents eight times to evaluate the solubility of the granules components in the NADES. Each of the treatments was done under similar conditions (time and temperature). After each treatment, the used NADES was collected for analysis, and freshly prepared NADES was added to the remaining granules. From the FEEM, it can be seen that each NADES solubilizes several components from the pilot-plant AGS. After the first treatment, humic-like matter (Region V) was present in the B:U:W (Fig. 10A1) and LA:Glc:W (Fig. 10B1), as well as microbial compounds (Region IV) for the LA:Glc:W and fluvic-like compounds (Region III) for B:U:W. Biological compounds are not observed after the first treatment of $\mathrm{B}: \mathrm{U}: \mathrm{W}$ but are observed in the fourth and eighth treatment. After the fourth (Fig. 10A2, B2) and eight (10A3, B3) treatments, the humic-like peaks started to have less intensity, and the microbial compounds (Region IV) began to have a higher intensity in the spectra. FEEM plots for the 2nd, 3rd, 5th, 6th, and 7th treatments can be found in SI (Figure S4). This result strongly suggests that the NADES are 
capable of solubilizing not only the proteins and polysaccharides from a complex biofilm such as the pilot-plant granules but also components of their matrix from their environment such as humic acids.

\section{Conclusions}

This study evaluated the potential of seven different NADES to be used as cleaning agents for biofouling by dissolving or breaking the biofilm structure. From the results of the studies we can conclude that:

- Two of the seven NADES tested (LA:Glc:W and B:U:W) successfully solubilized BSA, extracellular polymeric substances from a membrane biofilm, and extracted macromolecules (e.g., proteins) from the aerobic granular sludge.

- Both NADES, LA:Glc:W and B:U:W, have a strong impact on the granule shape, size, and structure. The NADES can substitute some of the granules' structural components, acting as an adhesive for the granules' remaining components.

- The treatment with B:U:W resulted in a weight reduction of the granule of $69 \%$ in the ATR-FTIR spectra, while the LA:Glc:W had a $48 \%$ weight reduction.

- The NADES B:U:W seems to be the more efficient NADES that can be used as a structural breaker since the granule's floccular shape and form are entirely lost.

- In a more complex matrix, as a pilot-plant granule, the NADES interact with the granule components by dissolving first the humiclike substances present in the granule moiety, followed by the solubilization of the biofilm macromolecules, which will affect the structure of the granules.

- This study presents NADES's potential to be used as a green solvent to weaken the biofilm structure and enhance its removal in systems such as membrane biofouling and to act as a green EPS extraction method for AGS.

\section{Declaration of Competing Interest}

The authors declare that they have no known competing financial interests or personal relationships that could have appeared to influence the work reported in this paper.

\section{Acknowledgment}

The authors thank King Abdullah University of Science and Technology (KAUST) for funding this research project.

\section{Supplementary materials}

Supplementary material associated with this article can be found, in the online version, at doi:10.1016/j.watres.2021.117323.

\section{References}

Beyer, F., Laurinonyte, J., Zwijnenburg, A., Stams, A.J.M., Plugge, C.M, 2017. Membrane fouling and chemical cleaning in three full-scale reverse osmosis plants producing demineralized water. J. Eng., 6356751

Chen, W., Westerhoff, P., Leenheer, J.A., Booksh, K., 2003. Fluorescence excitation Emission matrix regional integration to quantify spectra for dissolved organic matter. Environ. Sci. Technol. 37 (24), 5701-5710.

Choi, Y.H., van Spronsen, J., Dai, Y., Verberne, M., Hollmann, F., Arends, I.W., Witkamp, G.J., Verpoorte, R., 2011. Are natural deep eutectic solvents the missing link in understanding cellular metabolism and physiology? Plant Physiol. 156 (4), 1701-1705.

Creber, S.A., Vrouwenvelder, J.S., van Loosdrecht, M.C.M., Johns, M.L., 2010. Chemical cleaning of biofouling in reverse osmosis membranes evaluated using magnetic resonance imaging. J. Memb. Sci. 362 (1-2), 202-210.
Dai, Y.T., Rozema, E., Verpoorte, R., Choi, Y.H., 2016. Application of natural deep eutectic solvents to the extraction of anthocyanins from Catharanthus roseus with high extractability and stability replacing conventional organic solvents. J. Chromatogr. A 1434, 50-56.

Dai, Y.T., van Spronsen, J., Witkamp, G.J., Verpoorte, R., Choi, Y.H., 2013a. Natural deep eutectic solvents as new potential media for green technology. Anal. Chim. Acta 766, 61-68.

Dai, Y.T., Witkamp, G.J., Verpoorte, R., Choi, Y.H., 2013b. Natural deep eutectic solvents as a new extraction media for phenolic metabolites in Carthamus tinctorius L. Anal. Chem. 85 (13), 6272-6278.

Dai, Y.T., Witkamp, G.J., Verpoorte, R., Choi, Y.H., 2015. Tailoring properties of natural deep eutectic solvents with water to facilitate their applications. Food Chem. 187, 14-19.

Fang, C., Thomsen, M.H., Frankaer, C.G., Brudecki, G.P., Schmidt, J.E., AlNashef, I.M., 2017. Reviving Pretreatment Effectiveness of Deep Eutectic Solvents on Lignocellulosic Date Palm Residues by Prior Recalcitrance Reduction. Ind. Eng. Chem. Res. 56 (12), 3167-3174.

Farhat, N.M., Javier, L., Van Loosdrecht, M.C.M., Kruithof, J.C., Vrouwenvelder, J.S, 2019. Role of feed water biodegradable substrate concentration on biofouling: biofilm characteristics, membrane performance and cleanability. Water Res. 150, $1-11$.

Feng, C.J., Lotti, T., Lin, Y.M., Malpei, F., 2019. Extracellular polymeric substances extraction and recovery from anammox granules: evaluation of methods and protocol development. Chem. Eng. J. 374, 112-122.

Flemming, H.C., 2020. Biofouling and me: my Stockholm syndrome with biofilms. Water Res. 173, 115576.

Gonzalez, C.G., Mustafa, N.R., Wilson, E.G., Verpoorte, R., Choi, Y.H., 2018. Application of natural deep eutectic solvents for the "green"extraction of vanillin from vanilla pods. Flavour Fragr. J. 33 (1), 91-96.

Hall-Stoodley, L., Costerton, J.W., Stoodley, P., 2004. Bacterial biofilms: from the natural environment to infectious diseases. Nat. Rev. Microbiol. 2 (2), 95-108.

Kumar, A.K., Parikh, B.S., Pravakar, M., 2016. Natural deep eutectic solvent mediated pretreatment of rice straw: bioanalytical characterization of lignin extract and enzymatic hydrolysis of pretreated biomass residue. Environ. Sci. Pollut. R. 23 (10), 9265-9275.

Li, N., Wang, Y.Z., Xu, K.J., Huang, Y.H., Wen, Q., Ding, X.Q., 2016. Development of green betaine-based deep eutectic solvent aqueous two-phase system for the extraction of protein. Talanta 152, 23-32.

Lim, J.H., Song, S.H., Park, H.S., Lee, J.R., Lee, S.M., 2017. Spontaneous detachment of Streptococcus mutans biofilm by synergistic effect between zwitterion and sugar alcohol. Sci. Rep.-Uk 7.

Limoli, D.H., Jones, C.J., Wozniak, D.J., 2015. Bacterial extracellular polysaccharides in biofilm formation and function. Microbiol. Spectr. 3 (3).

Liu, Y., Friesen, J.B., McAlpine, J.B., Lankin, D.C., Chen, S.N., Pauli, G.F., 2018. Natural deep eutectic solvents: properties, applications, and perspectives. J. Nat. Prod. 81 (3), 679-690.

Ma, Y.J., Li, Y.R., Ali, S., Li, P.L., Zhang, W.Y., Rauch, M.C.R., Willot, S.J.P., Ribitsch, D., Choi, Y.H., Alcalde, M., Hollmann, F., Wang, Y.H, 2020. Natural deep eutectic solvents as performance additives for peroxygenase catalysis. ChemCatChem 12 (4), 989-994.

McSwain, B.S., Irvine, R.L., Hausner, M., Wilderer, P.A., 2005. Composition and distribution of extracellular polymeric substances in aerobic flocs and granular sludge. Appl. Environ. Microb. 71 (2), 1051-1057.

Nancharaiah, Y.V., Sarvajith, M., 2019. Aerobic granular sludge process: a fast growing biological treatment for sustainable wastewater treatment. Curr. Opin. Environ. Sci. Health 12, 57-65.

Paiva, A., Craveiro, R., Aroso, I., Martins, M., Reis, R.L., Duarte, A.R.C, 2014. Natural deep eutectic solvents - solvents for the 21st century. ACS Sustain. Chem. Eng. 2 (5), 1063-1071.

Plotka-Wasylka, J., Rutkowska, M., Owczarek, K., Tobiszewski, M., Namiesnik, J., 2017. Extraction with environmentally friendly solvents. Trac-Trends Analyt. Chem. 91, $12-25$.

Sanawar, H., Pinel, I., Farhat, N.M., Bucs, S.S., Zlopasa, J., Kruithof, J.C., Witkamp, G.J., van Loosdrecht, M.C.M., Vrouwenvelder, J.S., 2018. Enhanced biofilm solubilization by urea in reverse osmosis membrane systems. Water Res. X 1, 100004.

Vanda, H., Dai, Y.T., Wilson, E.G., Verpoorte, R., Choi, Y.H., 2018. Green solvents from ionic liquids and deep eutectic solvents to natural deep eutectic solvents. C.R. Chim. 21 (6), 628-638.

Vrouwenvelder, J.S., Bakker, S.M., Cauchard, M., Le Grand, R., Apacandie, M., Idrissi, M., Lagrave, S., Wessels, L.P., van Paassen, J.A., Kruithof, J.C., van Loosdrecht, M.C.M., 2007. The membrane fouling simulator: a suitable tool for prediction and characterisation of membrane fouling. Water Sci. Technol. 55 (8-9), 197-205.

Wani, T.A., Bakheit, A.H., Zargar, S., Hamidaddin, M.A., Darwish, I.A., 2017. Spectrophotometric and molecular modelling studies on in vitro interaction of tyrosine kinase inhibitor linifanib with bovine serum albumin. PLoS ONE 12 (4), e0176015.

Zhang, Q.H., Vigier, K.D., Royer, S., Jerome, F., 2012. Deep eutectic solvents: syntheses, properties and applications. Chem. Soc. Rev. 41 (21), 7108-7146.

Zhou, J., Wang, J.J., Baudon, A., Chow, A.T., 2013. Improved fluorescence excitationemission matrix regional integration to quantify spectra for fluorescent dissolved organic matter. J. Environ. Qual. 42 (3), 925-930. 